\title{
Entwicklung der genetischen und genomischen Medizin in der Schweiz
}

\begin{abstract}
Vorstand der SGMG: Isabel Filges ${ }^{a}$, Deborah Bartholdi ${ }^{b}$, Sven Cichon $^{c}$, Dunja Niedrist ${ }^{d}$, Naomi Porret $^{\mathrm{e}}$ Anita Rauch', Elisabeth Sallerg, Thomas von Känel ${ }^{\mathrm{h}}$, Siv Fokstuen ${ }^{\mathrm{i}}$

a PD Dr. med., Fachärztin für Medizinische Genetik, Spezialistin für medizinisch-genetische Analytik FAMH, Ärztliche Leiterin Medizinische Genetik, Institut für Medizinische Genetik und Pathologie, Universitätsspital und Universität Basel; ' PD Dr.med., Fachärztin für Medizinische Genetik, Oberärztin Humangenetik, Universitätsklinik für Kinderheilkunde, Inselspital Bern; ${ }^{c}$ Prof. Dr. sc. nat. Direktor Institut für Medizinische Genetik und Pathologie, Universitätsspital und Universität Basel; ${ }^{d}$ Dr. med., Fachärztin für Medizinische Genetik, Institut für Medizinische Genetik, Universität Zürich; ${ }^{e}$ Dr. sc. nat., Spezialistin für medizinisch-genetische Analytik FAMH, Laborleiterin Hämatologische Molekulare Diagnostik, Universitätsklinik für Hämatologie, Inselspital Bern;

f Prof. Dr. med., Fachärztin für Medizinische Genetik, Spezialistin für medizinisch-genetische Analytik FAMH, Ordinaria Institut für Medizinische Genetik, Universität Zürich; ${ }^{9}$ Dr. sc. nat., Spezialistin für medizinisch-genetische Analytik FAMH, Laborleiterin, Institut für medizinische \& molekulare Diagnostik AG, Falkenstrasse 14, 8024 Zürich; ${ }^{\text {h }}$ Dr. sc. nat., Spezialist für medizinisch-genetische Analytik FAMH, Chef du Service de Génétique Médicale, Hôpital du Valais, Sion, Co-Präsident SGMG; 'PD Dr. med., Fachärztin für Medizinische Genetik, Médecin adjointe agrégée, Service de Médecine Génétique, Département de médecine génétique, de laboratoire et de pathologie, Hôpitaux Universitaires de Genève, Co-Präsidentin SGMG
\end{abstract}

Mit den rasanten technologischen Entwicklungen im medizinisch-genomischen Bereich und dem damit verbundenen exponentiellen Kenntniszuwachs hat der Bedarf an genetischer Expertise in praktisch allen medizinischen Disziplinen zugenommen. Die Schweizerische Gesellschaft für Medizinische Genetik (SGMG) sieht sich als Fachgesellschaft und Vertreterin einer transversalen medizinischen Disziplin besonders geeignet, die gegebene Situation zu evaluieren und zukünftige Konzepte zu entwickeln und zu begleiten.

\section{Hintergrund und Herausforderungen}

Die SGMG verfolgt das Ziel, die Medizinische Genetik in Praxis, Lehre und Forschung zu fördern sowie hohe Standards in der klinisch-genetischen Patientenversorgung, Beratung und Diagnostik zu sichern [1]. Insbesondere in medizinisch-genetischen Zentren findet daher auch eine enge Zusammenarbeit mit den Fachspezialisten für Labormedizin (FAMH) der medizinischen Genetik statt.

Neben einem seit einiger Zeit signifikanten Zuwachs von Zuweisungen in die designierten Sprechstunden der Fachärzte für Medizinische Genetik erfolgen zahlreiche Anfragen von KollegInnen anderer Disziplinen

\section{Résumé}

Avec les développements technologiques fulgurants dans le domaine de la génomique médicale et l'accroissement exponentiel des connaissances dans ce domaine, le besoin d'expertise génétique a augmenté dans pratiquement toutes les spécialités médicales. La Société Suisse de Génétique Médicale (SSGM) se voit comme société de discipline médicale et représentante d'une spécialité médicale transversale, particulièrement adaptée à évaluer la situation actuelle, à développer de futurs concepts et à les accompagner. für konsiliarische und interdisziplinäre Mitbeurteilungen, nicht zuletzt auch wegen der zunehmenden Komplexität vieler genetischer Fragestellungen. Die Untersuchung vieler Gene gleichzeitig bis hin zum gesamten menschlichen Genom birgt neben den unbestrittenen Vorteilen viele Herausforderungen. Eine komplexere Indikationsstellung für genetische Untersuchungen, die Einordnung der Bedeutung von genomischen Varianten für die klinischen Befunde der Patienten sowie der Umgang mit unklaren Befunden und Zufallsbefunden seien hier als Beispiele genannt. Fragestellungen und Resultate in der genetischen und genomischen Medizin müssen vor allem auch bezüglich ihrer Bedeutung für andere Familienmitglieder und Nachkommen beurteilt werden (sogenannte transgenerational care). Viele genetische Laboruntersuchungen werden neben Fachärzten für Medizinische Genetik von Fachärzten anderer Disziplinen verordnet, die die Patienten hierzu beraten. Dies verlangt bei diesen eine fundierte Kenntnis der Möglichkeiten und Grenzen solcher Untersuchungen und der Interpretation der Ergebnisse. Eine Sicherung der Fortbildung ist hierfür unabdingbare Voraussetzung. Gemäss Gesetz über genetische Untersuchungen am Menschen (GUMG) werden die Veranlas- 
sung genetischer Untersuchungen und die genetische Beratung als ärztliche Verantwortung definiert. Insbesondere für pränatale wie auch präsymptomatische Fragestellungen wird eine entsprechende Weiterbildung gefordert [2]. Das BAG hat in der Analysenliste die mögliche Kostenübernahme von ausgedehnten Multi-GenPanels zunächst an eine Verordnung durch einen Facharzt für Medizinische Genetik geknüpft.

Von verschiedenen Seiten wird aktuell eine rege Debatte geführt, wie (in Zukunft) die erhöhten Herausforderungen der medizinisch-genetischen und genomischen Patientenversorgung gelöst werden sollen.

\section{Bestehende Konzepte}

Die Dachorganisation der SGMG, die European Society of Human Genetics (ESHG), und insbesondere deren European Board of Medical Genetics (EBMG), hat detaillierte "endorsed documents" zu den Kernkompetenzen und den Inhalten der Weiterbildung von Fachärzten für Medizinische (klinische) Genetik, Genetic Counsellors/ Genetic Nurses sowie Ärzten anderer Spezialisierungen mit genetischen Kompetenzen publiziert. Diese sind als europäischer Konsensus-Qualitätsstandard unter Berücksichtigung nationaler regulatorischer Unterschiede entstanden und anwendbar [3]. Die SGMG orientiert sich für die Schweiz an diesen Modellen, da sie eine fundierte Qualitätssicherung mit nachgewiesener Beteiligung medizinisch-genetischer Expertise garantieren.

Der Facharzt für Medizinische Genetik in der Schweiz entspricht den in Europa und international anerkannten und etablierten Anforderungen von Spezialisten für Medizinische (klinische) Genetik mit unbestrittenem Stellenwert in den Gesundheitssystemen. Verschiedene Fachgesellschaften, die die Notwendigkeit des Ausbaus genetischer Kenntnisse in ihrem Fachgebiet erkannt haben, organisieren in aktiver Zusammenarbeit und unter Teilnahme von Fachärzten für Medizinische Genetik vermehrt entsprechende Weiter- und Fortbildungsveranstaltungen. Mitglieder der SGMG sind somit bereits jetzt verstärkt in der Weiter- und Fortbildung der KollegInnen involviert. Die einzelnen Fortbildungsveranstaltungen sind meist auf den Homepages der Institute und der Fachgesellschaften einsehbar.

Formale interdisziplinäre Zusammenarbeiten zwischen der SGMG, anderen Fachgesellschaften und dem BAG sind aktuelle Erfolgsgeschichten der interdisziplinären Implementierung der genetischen Medizin. Zu nennen sind die Ausarbeitung des Expertenbriefs zur pränatalen Risikoabschätzung der häufigen fetalen Aneuploidien (Akademie fetomaternale Medizin, SGGG, SGMG), die Etablierung von Fortbildungsprogrammen und -boards speziell zur Präimplantationsdiagnostik in der Reproduktionsmedizin sowie die Sicherstellung der Qualität onkologisch-genetischer Beratungen durch Fachärzte für Onkologie und Medizinische Genetik gemeinsam mit der Schweizerischen Arbeitsgemeinschaft für Klinische Krebsforschung. Unserer Meinung nach ist dies der optimale Weg zu einer langfristigen professionellen Qualitätssicherung.

\section{Zukünftige Konzepte}

\section{Ärztliche Weiter- und Fortbildung}

Die SGMG unterstützt die Fortsetzung bereits bestehender Konzepte der ärztlichen Weiter- und Fortbildung in Medizinischer Genetik und plant eine engere Zusammenarbeit mit dem SIWF zu deren Sichtbarmachung sowie der Erweiterung und Formalisierung von genetischen Inhalten der Weiter- und Fortbildung in anderen medizinischen Fachdisziplinen. Die SGMG schlägt vor, sich hierfür an den von der ESHG/EBMG etablierten Modellen zu orientieren. Ein Angebot allgemeiner, in $\mathrm{Zu}$ sammenarbeit mit der SGMG erarbeiteter genetischer Kurse/CAS kann sicher auch helfen, der praktizierenden Ärzteschaft genetische und genomischen Grundkenntnisse zu vermitteln, die zu deren Studien- und Weiterbildungszeiten noch nicht verfügbar waren.

Ein allgemeiner Fähigkeitsausweis «Medizinische Genetik» wird von der SGMG nicht unterstützt. Fähigkeitsausweise beziehen sich in der Regel auf spezifische praktische Fertigkeiten, und nicht auf Kenntnisse und deren klinische Anwendung in einem individuellen Betreuungsprozess von Patienten. Da «Medizinische Genetik» ein eigener Facharzttitel darstellt und kein Teilfachgebiet, wird auch die Schaffung eines «Schwerpunkts Medizinische Genetik» von der SGMG nicht unterstützt. Gründe dafür sieht die SGMG vor allem im beträchtlichen Umfang des Fachgebietes der Medizinischen Genetik, das in einem "Facharzt light» nicht ausreichend vermittelt werden kann. Darüber hinaus bedarf der stetige rasante Erkenntnisgewinn in der Medizinischen Genetik einer kontinuierlichen und intensiven Fortbildung im Fach selbst.

Was die Ausbildung anbelangt, fördert die SGMG die aktuelle Initiative einzelner Fakultäten, verstärkt Konzepte der genetischen und genomischen Medizin in das Curriculum des Medizinstudiums zu integrieren, so dass absehbar die "genetic literacy» bei Ärzten im Allgemeinen steigen wird. Die SGMG ist der Ansicht, dass dies von allen Fakultäten in der Schweiz umgesetzt werden sollte.

\section{Konzepte für den klinischen Alltag}

In den universitären Spitälern, aber auch in nicht-universitären Kliniken gibt es regelmässige genetische 
Literatur

1 Filges und Miny. Schweiz Ärzteztg. 2009:90(21-22):844.

2 Gesetz über genetische Untersuchungen am Menschen, www.admin.ch

3 Core competencies in genetics for health professionals in Europe, https://www.eshg.org/ index.php?id=139.

Korrespondenz: PD Dr. med. Siv Fokstuen Fachärztin für Medizinische Genetik

Leitende Ärztin

Co-Präsidentin der SGMG

Service de médecine

génétique

Département de médecin génétique, de laboratoire

et de pathologie

Hôpitaux Universitaires

de Genève

Rue Gabrielle-Perret-Gentil 4

CH-1205 Genève

siv.fokstuen[at]hcuge.ch
Fallvorstellungen und Boards. Diese ermöglichen, vielschichtige Fragestellungen interdisziplinär in kollegialem Austausch zu lösen, und fördern auch die gegenseitige Expertise. Die SGMG erachtet eine vermehrte interdisziplinäre Zusammenarbeit im Rahmen von Boards sowie von gemeinsam durchgeführten Fallbesprechungen als eine sinnvolle Art, um regelmässige genetische Fortbildung in den klinischen Alltag zu integrieren und ihre Fachkompetenz einzubringen. Die SGMG ist der Meinung, dass solche Fallbesprechungen vermehrt auch für die in der Praxis tätigen Ärzte zugänglich sein sollten.

\section{Genetic Counsellors / Genetic Nurses}

In vielen Ländern mit einer etablierten medizinischgenetischen Patientenversorgung wird diese durch Genetic Counsellors unterstützt. Bisher ist dieses Berufsbild in der Schweiz, bis auf wenige kantonale Ausnahmen, nicht anerkannt, und es existiert keine formale Ausbildung. Einige im europäischen Ausland in akkreditierten Weiterbildungsprogrammen ausgebildete Genetic Counsellors unterstützen vor allem in der Romandie Abteilungen für Medizinische Genetik und haben sich in der ASCG (Association suisse des conseillers en génétique) vereinigt.

Entsprechend internationalem Vorbild unterstützt die SGMG die Tätigkeit von Genetic Counsellors, die an ein medizinisch-genetisches Zentrum angebunden sind und bei ihrer Tätigkeit unter Verantwortung und Supervision eines Facharztes für Medizinische Genetik stehen. Gemäss ESHG arbeitet ein Genetic Counsellor fokussiert auf spezifische genetische Erkrankungen, deren Diagnose bereits gestellt ist. Er/sie führt keine klinisch-diagnostischen Beurteilungen durch und ersetzt damit den Facharzt nicht. Als Aus- und Weiterbildungsstandard wird ein Masterstudiengang in international vergleichbarer Qualität angesehen, mit der entsprechenden Vermittlung von theoretischen und praktischen Kenntnissen in Zentren für Medizinische Genetik. Es wird erwartet, dass die praktischen Rotationen (etwa 50\% der Weiterbildung) in einem Zentrum für Medizinische Genetik stattfinden, dies unter Supervision eines erfahrenen Genetic Counsellors oder Facharztes für Medizinische Genetik. Die Etablierung eines solchen professionellen Studiengangs in der Schweiz müsste unter dem Gesichtspunkt des Weiterbildungsaufwands bei bestehenden Ressourcen und bei langfristigem Bedarf evaluiert werden. Alternativ könnte ein entsprechender ausländischer Abschluss anerkannt werden. Ein Nachteil wäre hier vermutlich für die deutschsprachige Schweiz zu erwarten, da im Moment kein deutschsprachiges Land die Weiterbildung anbietet. Letztendlich müssen auch für Genetic Counsel- lors zusätzliche Weiterbildungsstellen und Arbeitsplätze finanziert werden. Die Delegation von genetischen Beratungen an Personal, das nicht nach anerkannten Standards ausgebildet ist, unterstützt die SGMG nicht.

\section{Ressourcen}

Die zunehmende Zahl medizinisch-genetischer Konsultationen, die Sicherung und der Ausbau von Weiterund Fortbildungsveranstaltungen und -programmen, interdisziplinäre Zusammenarbeiten im klinischen Alltag gemeinsam mit anderen Fachdisziplinen sowie die eventuelle Planung einer zukünftigen Weiterbildung und/oder Supervision von Genetic Counsellors fordern personell vor allem auch die Fachärzte Medizinische Genetik. Die Entwicklung all dieser Aktivitäten und vor allem auch ihrer Qualitätssicherung durch Expertise wird nur dann möglich sein, wenn auch die Fachdisziplin der Medizinischen Genetik als solche gestärkt wird und die nötigen Ressourcen im Gesundheitssystem zur Verfügung stehen. Die Zahl der Fachärzte Medizinische Genetik sowie die Zahl der Weiterbildungs- und Kaderarztstellen in der Schweiz sind im Moment limitiert. Die SGMG sieht bei den gegebenen Herausforderungen Engpässe, wenn nicht auch für den Ausbau der personellen Ressourcen Sorge getragen wird. Hier sind insbesondere die Spitäler und universitären Fakultäten gefordert, sich für eine Stärkung einzusetzen, damit den anstehenden Ent wicklungen professionell Rechnung getragen werden kann.

\section{Fazit}

Die Entwicklungen und stetig wachsenden neuen Erkenntnisse sowie ihr interdisziplinärer und transversaler Ansatz machen die Medizinische Genetik zu einem äusserst faszinierenden Fachgebiet. Die zunehmende Bedeutung für zahlreiche medizinische Disziplinen verspricht spannende Zeiten in der weiteren Implementierung medizinisch-genetischer Kenntnisse in die Patientenversorgung. Deren Erfolg und Qualitätssicherung wird jedoch vor allem auch davon abhängig sein, ob die Fachdisziplin «Medizinische Genetik» selbst als Dreh- und Angelpunkt gestärkt wird. Die SGMG wird sich daher weiterhin für die Weiterbildung einer ausreichenden Zahl von Fachärzten für Medizinische Genetik einsetzen sowie gemeinsam mit dem SIWF ermöglichen, Kollegen anderer Fachdisziplinen neuestes Fachwissen in Weiter- und Fortbildung und interdisziplinären Boards zu vermitteln. Ziel ist die möglichst optimale Betreuung von Patienten und deren Familien mit einer genetischen Erkrankung. 